\title{
Effects of Training with Multi- Balls on Some Visual Abilities and Counter-Attack Skills for Junior Table Tennis Players.
}

\section{Sherif Fathy Saleh}

Racquet Sports, Sports Training Department. Faculty of Physical Education - Tanta University, Egypt.

\begin{abstract}
The current research aims at designing a recommended training program using multi-ball method and identifying its effects on some visual abilities and counter-attack skills of junior table tennis players. The researcher used the experimental approach (one-group design) with pre- and post-measurements. Participants $(n=15)$ were purposefully chosen from junior table tennis players less than 15 years of Gharbia Zone who are registered at the Egyptian Table Tennis Federation. Those players participated in Zones Champions Championship in Egypt. In the light of this research's aims, approach, sample, procedures and results, the researcher can conclude the following :

The recommended training program had significant effects on training junior table tennis players less than (15) year. ‘ Multi-ball training had positive effects on some visual abilities and counter-attack skills of junior table tennis players less than (15) years 'Counter-attack tests had positive effects on increasing the improvement percentage after applying the recommended program 'Here is a significant increase in players' abilities to use visual abilities and counter-attack skills (simple/ complex .(
\end{abstract}

Key words: Multi- Balls Training - Visual Abilities - Counter-attack Skills - Table Tennis.

\section{Introduction:}

$\mathrm{S}$ ports' training is an essential part of the sports preparation process as it is a physical and educational process based on using exercises to improve the physical, technical and tactical components necessary to achieve the best possible sports levels. Kennedy (2010) indicated the use of multiple training methods in addition to multiple load formations and rest intervals and concentration on specific sports physical, technical and psychological aspects for each sport (21:6).

Table tennis is a dynamic sport where training of sensory and motor systems affects performance. It requires unlimited technical and tactical skills. It can be classified as a fast ball sport as it requires anticipation, speed, reaction, powerful nervous system and decision making intelligence in all its levels (8: 24).

Multi-ball training is a new trend in table tennis. Swiss coaches use this type of training to improve the national teams' levels. The Chinese school used it to beat the German School as some experts call it Chinese fast balls. Tepper (2011) indicated that it depends on the coach as it is performed with a mass number of table tennis balls (50 -100). The coach hits the balls from the left side of the table and stands outside the table. He/she continues to hit balls with various speeds until it runs out or until the exercise duration is out. Balls are places in a basket near the table to be reached with the free hand of the coach. The coach varies racquet angle according to the skill under training (28: 166).
Turina (2006), Fullen (2011) and Letts (2013) indicated the advantages of multi-ball training as follows: intense performance in a very short time - saving time for collecting balls - performing strokes in a very short time increasing vital capacity and physical fitness - increasing or decreasing training load as needed - using different skills in the same framework .(6: 6) (22: 19) (10:15).

Wadea and Fekry (2002) indicated that multi-ball training has positive effects on aerobic and anaerobic endurance for athletes in addition to improving muscular strength, flexibility and agility. All these factors improve performance of table tennis. Furthermore, it affects the ability of cardio-pulmonary system positively to provide muscles with oxygen during performance.(29:132).

The researcher thinks that athletic improvement does not advance according to strict stages. Instead, it flows as an integrated process where the player can spend more or less time in any of its stages as needed. In addition, progress is not necessarily only forward, as the player may need to go back to previous stages to correct a problem of any sort.

Visual abilities represent a very important topic for many researchers, especially in table tennis as vision is extremely important for table tennis players where players depend greatly on several visual variables. A table tennis player should focus his/her vision on small ball with $40 \mathrm{~mm}$ of diameter and 2.7 grams in weight that moves in a free space without any clue for its direction. In addition, he/she should see the opponent and the table at the same time. Expert players can anticipate ball trajectory and the opponent's stroke, movement and pattern. This requires 
concentration and accuracy of performance. Williams (2012) indicated that only few studies dealt with how to modify visual abilities during acquiring skills. Therefore, this field needs more research (4: 170).

As a coach, chairman of coaches' committee and secretary of the scientific committee at ETTA, in addition to watching several national and international championships, the researcher thinks that table tennis players should fix vision on various distances and track balls with various speeds in changing visual environments. Visual skills should evolve well to improve these abilities as table tennis players need to see various objects outside the focus point, in front, to the side or even down. The player should distribute his/her eye-sight on side lines of the court and the final edge of the table as the player may lose the point if the ball exceeds that line. This requires peripheral eye-sight with visual tracking. This means that the player concentrates on the opponent's half-table and perceives the depth - distance between player and opponent - as this will help choosing the best counterattack and the speed of attack. Visual tracking means that the player tracks the opponent's body movements and footwork. This indicates the importance of training on accurate performance of attack and defense skills in addition to having the ability to direct the ball away from the opponent accurately and in time. Unfortunately, there are no previous research works, according to the researcher's knowledge, that used multi-ball training method in improving visual abilities for table tennis players.

The researcher thinks that multi-ball training is a strategic method that can be used in improving visual abilities and counter-attack skills for junior table tennis players as this will improve their real performance during matches.

Kondric Mrian et al (2007) indicated that any coach can judge elite table tennis players from their performance of attack and counter-attack using loop strokes with various spins to produce the required speed and spin (14: 393)

According to Baca Arnold (2007), Escobar-Vargas (2007), Hao Zhe (2007), Wang Yali (2007), Jili Song et al (2010) and Malagoli Lazoni et al (2010), attack tactics are now the dominant tactics in table tennis and this increased the chance to win the point during the first five balls in addition to increasing the offensive nature of the game. (5) (9) (11) (30) (13) (18).

This led the researcher to perform this study with the aim of designing a recommended training program using multi-ball method and identifying its effects on some visual abilities and counter-attack skills of junior table tennis players.

\section{Research Questions:}

Through designing a recommended training program using multi-ball method, the current research is trying to answer the following questions:

1. What are the effects of the recommended training program on some visual abilities of junior table tennis players less than 15 years?

2. What are the effects of the recommended training program on counter-attack skills of junior table tennis players less than 15 years?

\section{Hypotheses:}

1. There are statistically significant differences between the pre- and post-measurements of participants on performing some visual abilities of junior table tennis players less than 15 years.

2. There are statistically significant differences between the pre- and post-measurements of participants on performing counter-attack skills of junior table tennis players less than 15 years.

\section{Terminology:}

1. Visual abilities: it is a branch of measuring the sight field. It concentrates on vision as a perception, evaluation and improvement of visual performance (12: 22)

2. Counter-attack: it is the ability to attack against the opponent's attack using any type of spin with one or both faces of the racquet on the table surface to win the point (26: 108)

3. Table Tennis Robot: it is a device that allows the player to train without a partner with a builtin computer. It provides the chance to train on various speeds, altitudes, spins and directions as these variables cover all aspects of game situations. It has a remote-control function during play (27: 27).

\section{Review of Literature:}

Mohamed Abdalla (2011) performed a study titled by "Effects of a training program for visual skills on some visual abilities and service accuracy in tennis". The study aimed at designing a training program for qualitative visual skills and identifying its effects on some visual abilities like external awareness, visual tracking, hand-eye coordination and stroke accuracy. The researcher used the experimental approach on (30) students of faculty of physical education - Zagazig University as he divided the sample into two groups (15 students each). The researcher used ( $\mathrm{t}$ ) test and variance analysis. Results indicated that visual exercises had positive effects on visual abilities in 
addition to accuracy of straight, cut and twist serves in tennis. The regular exercises had positive effects on accuracy of straight, cut and twist serves without any effects on visual abilities (2: 40)

Sherif fathy saleh (2011) performed a study titled by "Designing and Calibrating Specific Tests for CounterAttack Skills in junior table tennis Players using Modified Electronic Ball Canon". The study aimed at designing a set of tests for counter-attack in junior table tennis players less than 18 years using the modified ball canon. The researcher used the descriptive approach on (112) junior table tennis players. The researcher used one way ANOVA for treating data. Results indicated that the designed tests are valid and highly reliable (26: 108-125).

Du Toit, PE Krüger, AF, Kleynhans, Jay-Du Preez, Govender (2011) performed a study titled by "The effects of sports exercises on the visual skills of university students". The study aimed at identifying the effects of some exercises on improving visual abilities. The researchers used the experimental approach on (169) students divided into two groups (control $=78-$ experimental $=91)$. The researcher used $(\mathrm{t})$ test and variance analysis. Results indicated that exercises can improve visual abilities like hand-eye coordination, visual sharpness, visualization and tracking. This in turn affects performance positively (7: 131).
According to the previous review of literature, it is clear that all studies were performed in 2011. Aims varied as one study aimed at designing a training program for qualitative visual skills and identifying its effects on some visual abilities like external awareness, visual tracking, hand-eye coordination and stroke accuracy while another study aimed at designing a set of tests for counter-attack in junior table tennis players less than 18 years using the modified ball canon and the third study aimed at identifying the effects of some exercises on improving visual abilities. All studies used either the descriptive or the experimental approach. Participant varied as some were students while others were junior athletes. None of these studies use the multi-ball method.

\section{Methods:}

\section{Approach:}

The researcher used the experimental approach (one-group design) with pre- and post-measurements.

\section{Participants:}

Participants $(n=15)$ were purposefully chosen from junior table tennis players less than 15 years of Gharbia Zone who are registered at the Egyptian Table Tennis Federation. Those players participated in Zones Champions Championship in Egypt. Table (1) indicates the descriptive data of participants.

Table (1):

Participants' homogeneity on all research variables $(n=15)$

\begin{tabular}{|c|c|c|c|c|c|c|}
\hline \multicolumn{2}{|r|}{ Variables } & Measurement & Mean & SD & Median & Squewness \\
\hline \multirow{3}{*}{$\begin{array}{l}\text { Growth } \\
\text { factors }\end{array}$} & Age & Year & 14.46 & 0.516 & 14.0 & 0.149 \\
\hline & Weight & $\mathrm{Kg}$ & 36.66 & 0.723 & 37.0 & 0.628 \\
\hline & Height & $\mathrm{Cm}$ & 134.06 & 0.798 & 134.0 & $0 . .128$ \\
\hline \multicolumn{2}{|r|}{ Training period } & Year & 5.446 & 0.5164 & 5.0 & 0.149 \\
\hline \multirow{6}{*}{$\begin{array}{l}\text { Physical } \\
\text { variables }\end{array}$} & Passing in $10 \mathrm{sec}$ & Number & 6.600 & 0.507 & 7.0 & 0.455 \\
\hline & Trunk leaning from standing & $\mathrm{Cm}$ & 6.600 & 0.5071 & 7.0 & 0.455 \\
\hline & Machine - pushed ball & Number & 10.666 & 0.488 & 11.0 & 0.788 \\
\hline & Accuracy of passing from motion & Number & 19.53 & 0.516 & 20.0 & 0.149 \\
\hline & Triangular jump & Number & 5.600 & 0.5071 & 0.600 & 0.045 \\
\hline & Pushing medicine ball $(1 \mathrm{~kg})$ & $\mathrm{Cm}$ & 18708 & 1.146 & 188.0 & 0.118 \\
\hline \multirow{4}{*}{$\begin{array}{c}\text { Visual } \\
\text { abilities }\end{array}$} & Peripheral eye sight & Point & 3.400 & 0.507 & 3.00 & 0.455 \\
\hline & Visual tracking & Point & 1.400 & 0.506 & 1.00 & 0.452 \\
\hline & Moving visual accuracy & Point & 1.333 & 0.488 & 1.00 & 0.788 \\
\hline & Hand-eye coordination & $\mathrm{Sec}$ & 8.333 & 0.487 & 8.00 & 0.789 \\
\hline \multirow{5}{*}{$\begin{array}{l}\text { Counter- } \\
\text { attack }\end{array}$} & Forehand Counter-attack against top spin strokes & Point & 12.400 & 0.507 & 12.00 & 0.455 \\
\hline & Forehand Counter-attack against side spin strokes & Point & 9.400 & 0.507 & 9.00 & 0.454 \\
\hline & Backhand Counter-attack against top spin strokes & Point & 7.400 & 0.508 & 7.00 & 0.456 \\
\hline & Smash counter-attack against smash strokes & Point & 9.333 & 0.488 & 9.00 & 0.788 \\
\hline & $\begin{array}{c}\text { Open (back hand/forehand) counter-attack against } \\
\text { back hand/forehand top- and side-spin }\end{array}$ & Point & 8.400 & 0.508 & 8.00 & 00.454 \\
\hline
\end{tabular}


Table (1) indicates that squewness ranged between $(0.45$ and 0.789) (between $3 \pm$ ). This indicates that values are free of radical distributions.

\section{The recommended training program:}

A) Objective: the program aims at training and improving some visual abilities and counter-attack skills for junior table tennis players less than (15) years.

B) Basics: the researcher considered the following:

1. Objectives of the program

2. Suitability of content to participants' abilities

3. Flexibility and applicability of content

4. Drills should be interesting

5. Gradual progression from easy to difficult and from simple to complex

6. Drills should be game-like

7. Intensity and volume increase should be gradual.

C) Content: the program includes technical drills. (Appendix 1) The training unit is divided into:

1- Warm-up: This part aims at preparing the player's body to effort. It divides into:

- General warm-up: to generally prepare the player's body to effort

- Specific warm-up: to prepare he player functionally, technically, tactically and mentally to effort. It should be individually tailored according to each player's abilities.
2- Main Part: This part includes drills (75-80\% of total duration of the unit). Duration of all contents is unified for all participants.

3- Drills: Through review of literature like Sherif Fathy Saleh (2008), Rowden Fullen (2011), Tepper Glenn (2011), Greg Letts (2013) and Ma Lin (2013), the researcher identified the suitable drills. (25: 2012) (22: 21) (28: 177) (10:18) (19:15).

4- Individual Lesson: This is the most important part of the training unit. The researcher applied these lessons during specific preparation and pre-competition periods. The researcher concentrated on counter-attack, footwork, and complex side, top and back spins. These lessons work on improving counter-attack through moves without ball or racquet, progression from stability to movement and from slow to fast, in addition to ball control and rally. The lessons also worked on progression from limited to open duration and beginning of rally (serve - receive - third ball). Lessons improve timing and tempo of play in addition to concentrating on first and third attack separately then combine them to win the point. Training situations should be game-like.

5- Training and competitive matches: These matches are very important as it provides an opportunity for players to apply all skills and tactics.

6- Cool Down: This is the final part of the unit and it aims at returning the player's body to normal.

Has been introduced elements of the proposed training program to a group of experts in the sport of table tennis has ranged percentage agreement between 85 to $100 \%$. (Appendix 2)

Table (2) shows experts' opinions about the contents of the recommended program.

Table (2):

experts' opinions about the contents of the recommended program

\begin{tabular}{|c|c|c|}
\hline Component & Experts' opinions & Agreement Percentage \\
\hline Program duration & 3 months & $100 \%$ \\
\hline Number of weeks & 12 weeks & $90 \%$ \\
\hline Number of units & 60 units & $90 \%$ \\
\hline Training days & 5 units per week & $95 \%$ \\
\hline Unit duration & $120 \mathrm{~min}$ & $95 \%$ \\
\hline Load formation & $1: 1-1: 2-1: 3$ & $85 \%$ \\
\hline Training loads & Light - moderate - sub-maximal & $90 \%$ \\
\hline Training method & $\begin{array}{ll} & \text { Multi-ball } \\
\text { - } & \text { High/low intensity intervals } \\
\end{array}$ & $100 \%$ \\
\hline Tests & $\begin{array}{ll} & \text { Visual abilities } \\
\text { - } & \text { Counter-attack } 5 \text { tests using Table Tennis Robot }\end{array}$ & $100 \%$ \\
\hline
\end{tabular}




\section{The researcher followed the following steps:}

1. Identifying the training period to be planned (preparation stage during 2013-2014 season)

2. Identifying number of units per week ( 5 units per week and each unit $=120$ minutes)

3. Identifying loads for each week as shown in figure (1)

Fig. (1):

load formation and number of units per week

\begin{tabular}{|c|c|c|c|c|c|c|c|c|c|c|c|c|}
\hline \multirow{2}{*}{ Loads } & \multicolumn{12}{|c|}{ Weeks } \\
\hline & 1 & 2 & 3 & 4 & 5 & 6 & 7 & 8 & 9 & 10 & 11 & 12 \\
\hline \multicolumn{13}{|c|}{ Sub-maximal $(75-80 \%)$} \\
\hline \multicolumn{13}{|c|}{ Moderate $(50-75 \%)$} \\
\hline \multicolumn{13}{|c|}{ Light $(40-50 \%)$} \\
\hline Units & 5 & 5 & 5 & 5 & 5 & 5 & 5 & 5 & 5 & 5 & 5 & 5 \\
\hline Formation & $1: 1$ & $1: 1$ & $1: 1$ & $1: 2$ & $1: 2$ & $1: 2$ & $1: 1$ & $1: 2$ & $1: 3$ & $1: 3$ & $1: 3$ & $1: 3$ \\
\hline
\end{tabular}

Rest intervals and pulse recovery rate for each player were calculated according to training loads. Pulse rate were calculated during 6 seconds. Training loads were controlled through changing intensity and stabilizing volume and rest intervals. Intensity was controlled through changing the sequence of ball throws, velocities and altitudes over the net in addition to the distance between player and table. Moderate intensity was set at $35 \mathrm{balls} / \mathrm{minute}$ and $3 \mathrm{~m}$ distance. Sub-maximal intensity was set at 50 balls/minute and $2 \mathrm{~m}$ distance. Maximal intensity was set at $70 \mathrm{balls} / \mathrm{minute}$ and $1 \mathrm{~m}$ distance.

Table (3) shows percentages of content and durations for physical, technical and tactical preparation.

Table (3):

percentages of preparation content

\begin{tabular}{|c|c|c|c|c|c|c|}
\cline { 2 - 6 } \multicolumn{1}{c|}{} & \multirow{2}{*}{$\begin{array}{c}\text { Physical } \\
\text { preparation }\end{array}$} & $\begin{array}{c}\text { Technical } \\
\text { preparation }\end{array}$ & \multicolumn{2}{c|}{ Tactical preparation } & \multirow{2}{*}{ Cool down } & \multirow{2}{*}{ Total } \\
\cline { 3 - 6 } & Technical drills & Tactics & $\begin{array}{c}\text { Competitive } \\
\text { matches }\end{array}$ & & \\
\hline Percentages & $20 \%$ & $25 \%$ & $25 \%$ & $25 \%$ & $5 \%$ & $100 \%$ \\
\hline Units & $1440 \mathrm{~min}$ & $1800 \mathrm{~min}$ & $1800 \mathrm{~min}$ & $1800 \mathrm{~min}$ & $360 \mathrm{~min}$ & $7200 \mathrm{~min}$ \\
\hline Unit & $24 \mathrm{~min}$ & $30 \mathrm{~min}$ & $30 \mathrm{~min}$ & $30 \mathrm{~min}$ & $6 \mathrm{~min}$ & $120 \mathrm{~min}$ \\
\hline
\end{tabular}

Table (3) indicates that duration of psychological and mental preparations is not calculated among the total duration of training units as most of this preparation is done outside the training unit. In table tennis, technical preparation is mixed with tactical preparation in one unit as tactics are the application of skills with all its physical and mental requirements on various places of the table.

Data collection tools:

\section{- The researcher used the following tools:}

1- Physical tests

2- Visual

abilities tests

3- Counter-attack tests (by 4- Personal data logs the researcher)

5- Medical balance for 6- Blue table tennis measuring weights

tables

7- Table tennis robot

8- Legal

(ITTF approved) nets

9- Legal White and yellow "Butterfly" balls (3 stares) with $40 \mathrm{~mm} / \mathrm{meter}$ and $2.7 \mathrm{~g}$ in weight

- The researcher used the following physical tests: (Appendix 3)

1- Passing in $10 \mathrm{sec}$

(speed)

2- Trunk leaning from (flexibility) standing

3- Machine - pushed ball

(racquet hand reaction) 
4- Accuracy of passing from (accuracy) motion

5- Triangular jump

(agility)

6- Pushing medicine ball (1 (speed $\mathrm{kg})$

(1:230-236)

-The researcher used the following visual abilities tests: (Appendix 4)

1- Peripheral eye sight

Visual tracking

3- Moving visual accuracy

Hand-eye coordination (24:626)

- The researcher used the following counter-attack tests from design researcher: (Appendix 5)

$2-$ 4-
1- Forehand Counter-attack against top spin strokes

2- Forehand Counter-attack against side spin strokes

3- Backhand Counter-attack against top spin strokes

4- Smash counter-attack against smash strokes

5- Open (back hand/forehand) counter-attack against back hand/forehand top- and side-spin. (26: 108-125)

Validity and Reliability of Tests:

To calculated validity and reliability of tests, the researcher a[[lied these tests to a pilot sample $(n=12)$ from Ghazl Al-Mahalla Sports Club from 5-7-2013 to 6-7-2013. Sample was divided into two group (distinguished = nondistinguished $=6$ ). Table (4) shows variance significance between the two groups for tests validity.

Table (4):

variance significance between the distinguished and non-distinguished groups on all tests $(\mathrm{n}=12)$

\begin{tabular}{|c|c|c|c|c|c|c|c|}
\hline & \multirow[t]{2}{*}{ Variables } & \multirow[t]{2}{*}{ Measurement } & \multicolumn{2}{|c|}{$\begin{array}{l}\text { Distinguished } \\
\quad(\mathrm{n}=6)\end{array}$} & \multicolumn{2}{|c|}{$\begin{array}{c}\text { Non- } \\
\text { distinguished } \\
(n=6)\end{array}$} & \multirow[t]{2}{*}{$(\mathrm{T})$} \\
\hline & & & Mean & SD & Mean & SD & \\
\hline \multirow{6}{*}{$\begin{array}{l}\text { Physical } \\
\text { variables }\end{array}$} & Passing in $10 \mathrm{sec}$ & Number & 7.625 & 0.517 & 3.750 & 0.707 & $* 121.05$ \\
\hline & Trunk leaning from standing & $\mathrm{Cm}$ & 8.625 & 0.517 & 4.62 & 0.518 & $* 15.45$ \\
\hline & Machine - pushed ball & Number & 13.625 & 0.517 & 8.5 & 0.534 & $* 19.48$ \\
\hline & Accuracy of passing from motion & Number & 22.37 & 0.518 & 16.75 & 0.462 & $* 22.91$ \\
\hline & Triangular jump & Number & 7.37 & 0.517 & 3.75 & 0.463 & $* 14.76$ \\
\hline & Pushing medicine ball (1 kg) & $\mathrm{Cm}$ & 165.37 & 0.516 & 160.3 & 0.517 & $* 19.32$ \\
\hline \multirow{4}{*}{$\begin{array}{c}\text { Visual } \\
\text { abilities }\end{array}$} & Peripheral eye sight & Point & 5.333 & 0.516 & 3.333 & 0.514 & $* 3.708$ \\
\hline & Visual tracking & Point & 3.50 & 0.547 & 1.66 & 0.516 & *5.966 \\
\hline & Moving visual accuracy & Point & 3.500 & 0.547 & 0.666 & 0.514 & $* 9.220$ \\
\hline & Hand-eye coordination & $\mathrm{Sec}$ & 6.666 & 0.516 & 10.50 & 0.547 & $* 12.474$ \\
\hline \multirow{5}{*}{$\begin{array}{l}\text { Counter } \\
\text { attack }\end{array}$} & Forehand Counter-attack against top spin strokes & Point & 16.666 & 0.517 & 7.500 & 0.549 & $* 29.82$ \\
\hline & $\begin{array}{c}\text { Forehand Counter-attack against side spin } \\
\text { strokes }\end{array}$ & Point & 13.66 & 0.516 & 5.500 & 0.548 & $* 26.57$ \\
\hline & $\begin{array}{c}\text { Backhand Counter-attack against top spin } \\
\text { strokes }\end{array}$ & Point & 11.50 & 0.547 & 5.66 & 0.516 & $* 18.98$ \\
\hline & Smash counter-attack against smash strokes & Point & 14.66 & 0.516 & 7.166 & 0.408 & $* 27.90$ \\
\hline & $\begin{array}{l}\text { Open (back hand/forehand) counter-attack } \\
\text { against back hand/forehand top- and side-spin . }\end{array}$ & Point & 11.50 & 0.546 & 6.000 & 0.632 & $* 16.10$ \\
\hline
\end{tabular}

(t) Table value on $P \leq 0.05=2012$

Table (4) indicates statistically significant differences between the distinguished and non-distinguished groups in favor of the distinguished group. This indicates tests validity.

As for reliability, the researcher used test/retest procedures with time interval of (3) days on a pilot sample (n=6) from Ghazl Al-Mahalla Sports Club. Table (5) shows results. 
Table (5):

correlation coefficients for all tests $(n=6)$

\begin{tabular}{|c|c|c|c|c|c|c|c|}
\hline \multirow{2}{*}{\multicolumn{2}{|c|}{ Variables }} & \multirow{2}{*}{ Measurement } & \multicolumn{2}{|c|}{ Test } & \multicolumn{2}{|c|}{ Retest } & \multirow{2}{*}{ (R) } \\
\hline & & & Mean & SD & Mean & SD & \\
\hline \multirow{6}{*}{$\begin{array}{l}\text { Physical } \\
\text { variables }\end{array}$} & Passing in $10 \mathrm{sec}$ & Number & 7.625 & 0.517 & 7.375 & 0.744 & $* 0.788$ \\
\hline & Trunk leaning from standing & $\mathrm{Cm}$ & 8.625 & 0.517 & 8.437 & 0.728 & $* 0.876$ \\
\hline & Machine - pushed ball & Number & 13.625 & 0.517 & 13.33 & 0.715 & $* 0.777$ \\
\hline & Accuracy of passing frommotion & Number & 22.37 & 0.518 & 22.12 & 0.640 & $* 0.868$ \\
\hline & Triangular jump & Number & 7.37 & 0.517 & 7.15 & 0.798 & $* 0.882$ \\
\hline & Pushing medicine ball (1 kg) & $\mathrm{Cm}$ & 165.37 & 0.516 & 165.1 & 0.834 & $* 0.868$ \\
\hline \multirow{4}{*}{$\begin{array}{l}\text { Visual } \\
\text { abilities }\end{array}$} & Peripheral eye sight & Point & 5.333 & 0.516 & 5.450 & 0.571 & $* 0.928$ \\
\hline & Visual tracking & Point & 3.500 & 0.547 & 3.616 & 0.491 & $* 0.854$ \\
\hline & Moving visual accuracy & Point & 3.500 & 0.547 & 3.650 & 0.550 & $* 0.896$ \\
\hline & Hand-eye coordination & $\mathrm{Sec}$ & 6.666 & 0.516 & 6.816 & 0.457 & $* 0.874$ \\
\hline \multirow{5}{*}{$\begin{array}{l}\text { Counter- } \\
\text { attack }\end{array}$} & $\begin{array}{c}\text { Forehand Counter-attack against loop top spin } \\
\text { strokes }\end{array}$ & Point & 16.66 & 0.517 & 16.83 & 0.516 & $* 0.875$ \\
\hline & $\begin{array}{l}\begin{array}{l}\text { Forehand Counter-attack against side spin } \\
\text { strokes }\end{array} \\
\end{array}$ & Point & 13.66 & 0.516 & 13.81 & 0.457 & $* 0.874$ \\
\hline & $\begin{array}{c}\text { Backhand Counter-attack against top spin } \\
\text { strokes }\end{array}$ & Point & 11.500 & 0.547 & 11.68 & 0.775 & $* 0.865$ \\
\hline & Smash counter-attack against smash strokes & Point & 14.66 & 0.516 & 14.86 & 0.463 & $* 0.847$ \\
\hline & $\begin{array}{c}\text { Open (back hand/forehand) counter-attack } \\
\text { against back hand/forehand top- and side-spin . }\end{array}$ & Point & 11.50 & 0.546 & 11.63 & 0.496 & $* 0.809$ \\
\hline
\end{tabular}

\section{Significance on $0.05=0.666$}

Table (5) indicates statistically significant correlations between test and retest on all tests used. This indicates reliability of tests.

\section{Main Experiment:}

\section{Pre-measurements:}

After calculating validity and reliability of tests, premeasurements were taken from 11-7-2013 to 12-7-2013.

Main application:
The recommended program was applied from 13-7-2013 to 3-10-2013 in the table tennis hall - Tanta Sports Club.

Post-measurements:

Post-measurements were taken after the program from 510-2013 to 6-10-2013.

Statistical Treatment:

The researcher used SPSS software to calculate the following: mean - median - SD - (t) test - squewness correlation coefficients - improvement percentages. 


\section{Results:}

Table (6):

difference significance between pre- and post-measurements of the experimental group on visual abilities tests $(\mathrm{n}=15)$

\begin{tabular}{|c|c|c|c|c|c|c|c|c|}
\hline & \multirow{2}{*}{ Variables } & \multicolumn{2}{|c|}{ Pre- } & \multicolumn{2}{|c|}{ Post- } & \multirow{2}{*}{$\begin{array}{c}\text { Means } \\
\text { Difference }\end{array}$} & \multirow{2}{*}{ (t) } & \multirow{2}{*}{ Improvement (\%) } \\
\hline & & Mean & SD & Mean & SD & & & \\
\hline \multirow{4}{*}{$\begin{array}{c}\text { Visual } \\
\text { abilities }\end{array}$} & Peripheral eye sight & 3.400 & 0.507 & 8.40 & 0.736 & 5.00 & 19.365 & $\% 147.04$ \\
\hline & Visual tracking & 1.400 & 0.506 & 4.60 & 0.507 & 3.200 & 18.330 & $\% 228.5$ \\
\hline & Moving visual accuracy & 1.333 & 0.488 & 4.73 & 0.457 & 3.40 & 25.968 & $\% 255.06$ \\
\hline & Hand-eye coordination & 8.333 & 0.487 & 6.60 & 0.507 & 1.733 & 8.404 & $\% 20.80$ \\
\hline
\end{tabular}

(t) Table value on $P \leq 0.05=2.14$

Table (6) indicates statistically significant differences between pre- and post-measurements of visual abilities in favor of the post-measurements.

Table (7):

difference significance between pre- and post-measurementsof the experimental group on counter-attack tests $(\mathrm{n}=15)$

\begin{tabular}{|c|c|c|c|c|c|c|c|c|}
\hline \multirow{2}{*}{\multicolumn{2}{|c|}{ Variables }} & \multicolumn{2}{|c|}{ Pre- } & \multicolumn{2}{|c|}{ Post- } & \multirow{2}{*}{$\begin{array}{c}\text { Means } \\
\text { Difference }\end{array}$} & \multirow{2}{*}{ (t) } & \multirow{2}{*}{$\begin{array}{c}\text { Improvement } \\
(\%)\end{array}$} \\
\hline & & Mean & SD & Mean & SD & & & \\
\hline \multirow{5}{*}{ 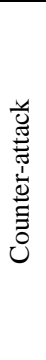 } & $\begin{array}{l}\text { Forehand Counter-attack against } \\
\text { top spin strokes }\end{array}$ & 12.40 & 0.507 & 26.2 & 0.774 & 13.80 & 79.04 & $\% 111.2$ \\
\hline & $\begin{array}{l}\text { Forehand Counter-attack against } \\
\text { side spin strokes }\end{array}$ & 9.40 & 0.507 & 21.46 & 0.516 & 12.06 & 66.40 & $\% 128.2$ \\
\hline & $\begin{array}{c}\text { Backhand Counter-attack against } \\
\text { top spin strokes }\end{array}$ & 7.40 & 0.508 & 13.46 & 0.639 & 6.066 & 29.41 & $\% 81.97$ \\
\hline & $\begin{array}{c}\text { Smash counter-attack against } \\
\text { smash strokes }\end{array}$ & 9.33 & 0.488 & 2306 & 0.593 & 13.73 & 75.58 & $\% 147.1$ \\
\hline & $\begin{array}{c}\text { Open (back hand/forehand) } \\
\text { counter-attack against back } \\
\text { hand/forehand top- and side-spin }\end{array}$ & 8.40 & 0.508 & 20.13 & 0.639 & 11.73 & 64.57 & $\% 139.6$ \\
\hline
\end{tabular}

(t) Table value on $P \leq 0.05=2.14$

Table (7) indicates statistically significant differences between pre- and post-measurements of counter-attack in favor of the post-measurements.

\section{Discussion:}

Table (6) indicates statistically significant differences between pre- and post-measurements of some visual abilities of junior table tennis players less than (15) years in favor of the post-measurements.

The researcher thinks this improvement is due to the efficiency of the recommended training program that included technical drills suitable for this age group, in addition to multi-ball training. Table tennis depends on the ability to respond to overlapping variables during matches. Multi-ball training allows this to happen and using probability theory, we can make new training patterns that enrich the training process.

Alessandro et al (2011) indicated that training program should improve performance in table tennis as these programs represent the only guarantee for progress in this sport, especially during competitions (3: 27).

In addition, table (6) indicates that improvement percentages for peripheral eye sight, visual tracking, moving visual accuracy and hand-eye coordination were $147.04 \%, 228.5 \%, 225.06 \%$ and $20.820 \%$ respectively. This is due to the fact that players developed experiences from the program that allowed them to anticipate the most probable variables.

Drills with various directions, speeds, ball launching angles and ball drops on the table with all types of top, side, back and no spin had positive effects. Multi-ball training played a major role in increasing the improvement percentage of visual abilities as it enabled players to control the racquet angles and directions. It also stimulated the visual nerve and this led to more accurate notice of the balls coming from the coach or the opponent.

Sebastian Schwab \& Daniel Memmert (2012) indicated that training programs can improve eye sight for male 
racquet sports players between 12 and 16 years of age after (6) weeks of training. The recommended program lasted for (12) weeks and targeted players less than (15) years of age. Crognier et al (2003) indicated that players depend on their previous knowledge tactics. (24: 629) (16: 123).

Improvement percentage of hand-eye coordination reached $20.820 \%$ as players made decisions taking less time than allowed. This is an advantage in performing successful strokes. It is also noteworthy that some systematic differences in peripheral eye sight appeared among players as they preferred to concentrate on information that is more vivid and specific from the areas between the ball and racquet. Players used the complex skill of visual tracking as they used previous experiences to direct vision towards additional information that may be more stable for the task at the area around the head, shoulder, trunk and thigh. Players also depended on late information in moving visual accuracy as this type of information is of affirmative nature to them.

This is consistent with Row \& McKenna (2011) who indicated that training of visual abilities is dealt with through: reading the movements of the ball and opponent, recalling moves used in previous situations and identifying the distinct features of the opponent (23: 62).

E.Jadehpur \& Yarigholi (2004) indicated that improving adaptation and sight sharpness may improve the vision system. This leads to a decrease in time of gaining vivid picture. In table tennis, the focus point changes as fast as the change of ball and opponent speed. Balls move with high velocities the eye should see it vividly. Therefore, the player should follow the ball as fast as possible to perform successfully. Pop up movement of the eye may be used to focus on the ball. This movement may be blocked so that what can be seen is only the first and last fixed points while the whole trajectory is blocked. Therefore, these two particular points are very important. The player should change his/her adaptation as fast as the eye moves so that objectives can be achieved during play. When peripheral eye sight is improved moving visual accuracy is improved too (8: 28).

Kondric Mrian (2013) indicated that indicated that improving moving visual accuracy helps not only relaxation of eye muscles, but also works on strengthening muscles behind the eye .

This is consistent with Du Toit et al (2001) in that sports drills can improve some visual abilities and this leads to improving motor skills, cognitive learning and performance (7: 134).
This proves the first hypothesis stating that: "There are statistically significant differences between the preand post-measurements of participants on performing some visual abilities of junior table tennis players less than 15 years".

Table (7) indicates statistically significant differences between pre- and post-measurements of junior table tennis players less than (15) years on counter-attack in favor of the post-measurements.

The researcher thinks this improvement is due to the efficiency of the recommended training program that included technical drills suitable for this age group, in addition to multi-ball training. Table tennis depends on the ability to respond to overlapping variables during matches. Evaluating the performance level of counter-attack should be according to international law amendments and the game requirements of velocities, spins, altitudes and directions. Players should be good at counter-attack for all distances and racquet/table angles.

L. N. Xu (2013) indicated that multi-ball training is a very important part of the training program of the Chinese national team (world champions of table tennis). It is the reason behind maintaining China as a pioneer of table tennis and cope up with modern technological advances and innovations (17: 22).

In addition, table (7) indicates that improvement percentages for Forehand Counter-attack against top spin strokes, Forehand Counter-attack against side spin strokes, Backhand Counter-attack against top spin strokes, Smash counter-attack against smash strokes and Open (back hand/forehand) counter-attack against back hand/forehand top- and side-spin are $111.29 \%, 128.29 \%, 81.97 \%$, $147.14 \%$ and $139.64 \%$ respectively.

The researcher thinks that this improvement is due to the components of the recommended program as it included various aspects that aimed at improving the performance level of counter-attack using game-like situations and various drills to avoid boredom.

The researcher concentrated on footwork through tasks and training situations like short steps (sliding swinging), pivot steps (one step - one side to the other), deep steps (front/back running) and long steps (one side to the other - crossing) in relatively short durations, in addition to repeating footwork to maintain tempo.

Through multi-ball training, the researcher concentrated on footwork drills and counter-attack from short, medium and long distances from the table in addition to linking counter-attack to footwork like putting the foot sideways in the ball direction before and after racquet move according to the available time. The researcher also 
concentrated on forward/backward runs with short steps backward, forward then backward to improve running speed. In addition, footwork, effects of the opponent's top spin and directing balls away from the opponent are all linked to Forehand Counter-attack against top spin strokes, Forehand Counter-attack against side spin strokes and Backhand Counter-attack against top spin strokes. Multiball training is based on game-like situations.

Improvement in these variables is due to multi-ball training with all its variables (velocity - spin - altitude rally) as these variables helped the researcher to design the training program in addition to increasing players' active involvement in training and providing them with confidence.

Qu Wang (2013) and X. W. Xiang (2013) agreed that multi-ball training is characterized with speed. This is clear in footwork drills and all types of defense including counter-attack from various distances and sudden attack from far distances and defensive situations. (20: 3) (31: 4).

This proves the second hypothesis stating that: "There are statistically significant differences between the preand post-measurements of participants on performing counter-attack skills of junior table tennis players less than 15 years".

\section{Conclusions:}

In the light of this research's aims, approach, sample, procedures and results, the researcher can conclude the following:

1. The recommended training program had significant effects on training junior table tennis players less than (15) years.

2. Multi-ball training had positive effects on some visual abilities of junior table tennis players less than (15) years.

3. Multi-ball training had positive effects on some counter-attack skills of junior table tennis players less than (15) years.

4. Counter-attack tests had positive effects on increasing the improvement percentage after applying the recommended program.

5. Here is a significant increase in players' abilities to use visual abilities and counter-attack skills (simple/ complex).

\section{Recommendations:}

In the light of these research conclusions, the researcher recommends the following:
1. Applying the recommended training program using multi-ball approach in training junior table tennis players less than (15) years

2. Using multi-ball approach to improve visual abilities due to its positive effects in table tennis

3. Using multi-ball approach to improve counterattack skills due to its positive effects in table tennis

4. Using multi-ball approach to help junior table tennis in individual and team training

5. Using results of this research in planning training programs

6. Other research works should deal with other aspects of multi-ball training as it relates to technical and tactical preparation

7. Performing similar studies to identify the effects of multi-ball training on defensive aspects of table tennis .

\section{References:}

1. Abdalla, Mohamed AScientific bases in table tennis and measurement methods. Ayat Press - Zagazig, 2007, Egypt (in Arabic)

2. -------Effects of a training program for visual skills on some visual abilities and service accuracy in tennis. Scientific Journal for Physical Education and Sport, No, 42, 2011 - Faculty of Physical Education for Women - Alexandria University, Egypt .

3. Alessandro M. Zagatto, Marcelo Papoti \& Claudio Ao Comparison of anaerobic threshold, oxygen uptake and heart rate between specific table tennis procedure and conventional ergometers, International Journal of Table Tennis Sciences, No.7, 2011.

4. A. Mark WilliamsVisual Search Behaviour In Sport, Journal of Sports Sciences, Vol. 40, 2012 .

5. Baca Arnold

6. Qualitative game analysis in table Tennis,10th Anniversary ITTF Sports Science Congress, University of Zagreb, Croatia, May, 2007.

7. Boris Turina Some Advice Concerning Multi Ball training, http://www.masatenisi.org/english/multiball.htm, 2006.

8. Du Toit, PE Krüger, AF, Kleynhans, Jay-Du Preez, Govender,The effects of sports exercises on the visual skills of university students, African Journal for Physical, Health Education, Recreation and Dance ,http://www.ajol.info/index.php ,Vol 17, No 3 ,2011.

9. Ebrahim Jafarzadehpur \& Mohammad R. Y Comparison of Visual Acuity in Reduced Lamination and Facility of Ocular Accommodation in Table Tennis Champions and 
Non- Players, Journal of Sports Science and Medicine, http://www.jssm.org, 2004.

10. Escobar-Vargas Jorge

1. Notational analysis for competition in table Tennis (part I): based Format analysis, 10th Anniversary ITTF Sports Science Congress, University of Zagreb, Croatia, May, 2007.

11. greg Letts Multi ball Table Tennis Training - Using Multiball in Your Ping-Pong Practice http://tabletennis.about.com/od/trainin 1/a/multiball,20 13 .

12. Hao, Zhe

1. Analysis on technic and tactics of Ryu Seung-Min in man's singles table tennis final and semifinal of the 28th Olympic games in Athens, 10th Anniversary ITTF Sports Science Congress, University of Zagreb, Croatia, May, 2007.

13. Isabel Walker Why visual training programmers for sport don't work, peak performance, www.pponline.co.uk, 2001.

14. Jili Song, Zhe Hao, Zhensheng Tian, Yujiao Hao Analysis on Technique and Tactics of Lin Ma and Hao Wang in the Men's Single Table Tennis Final in the 29th Olympic Games ,11 th ITTF sports Science Congress in Japan http://www.jtta.or.jp/11th_ittf_ssc, April 2010 .

15. Kondric, Miran Supej, Matej, Nemec, Bojan, Hudetz, Radivoj, Koscak, Ju Possibilities to reduce speed and spin by

16. changing the thickness of sponge, upper layer or total thickness of sandwich Rubber, 10th Anniversary ITTF Sports Science Congress, University of Zagreb, Croatia, May, 2007.

17. Kondric, Miran Planning with a Difference for World Hopes Team, a Scientific Approach, http://www.ittf.com/ front page/ittf,2013

18. L. Crognier1, Y.A. Féry and N. Veret Is anticipation in tennis related to specific knowledge, 8th World Congress of Science and Racket Sports France, May, 2003.

19. $\mathrm{L} \mathrm{NXu}$ Multi-ball Training Method In Table Tennis Technical And Tactical Application Of Combination, http://www.globethesis.com, 2013 .

20. Malagoli Lanzoni Ivan, Lobietti Roberto, Merni Fran Footwork in Relationship with Strokes and Efficacy during the 29th Olympic Games Table Tennis Final, international Journal of Table Tennis Sciences , No.6, 2010 .

21. Ma Lin, Ma Lin Training Multi-Ball, http://www.tabletennisdb.com/videos/ma-lin-trainingmulti-ball.html ,2013.
22. QuWang: Experimental Study On "Single And Multiball Combination" Teaching Method In Table Tennis Elective Course Of University,

23. http://, www.globethesis.com, 2013 .

24. Robert Kennedy Circuit Training, University of Cumbria Online, https://college.ch/robot,2010.

25. Rowden fullen The Way The Chinese Use Multi-Ball, http://protabletennis.net/content/waychinese-use-multi-ball,2011.

26. Rowe, R. M., \& McKenna, F. P Skilled anticipation in real-world tasks: Measurements of attentional demands in the domain of tennis, Journal of Experimental Psychology, 2011.

27. Sebastian Schwab\& Daniel Memmert The impact of a sports vision training program in youth field hockey players, Journal of Sports Science and Medicine, No. 11, 2012.

28. Sherif fathy salehDesigning curricula as interactive CDs. E-Learning Center - Tanta University, 2008. www.tanta-elearning.com (in Arabic)

29. ----------- Designing and Calibrating Specific Tests for Counter-Attack Skills in junior table tennis Players using Modified Electronic Ball Canon, Theories\& Applications, the International Edition(TIAE), Faculty of Physical Education ,Abu Qir-Alexandria University ,2011.

30. Tamasu Butterfly Amicus 3000 Plus table tennis robot, www.butterfly-world.com , 2009.

31. Tepper Glenn

1. Table Tennis ITTF Advanced Coaching Manual, Shanghai Minsun, Oct, 2011.

32. Wadea, Elin \& Fekg, Salwa Reference in table tennis, training - teaching, Monshaat Al-Maaref Alexandria - Egypt, 2002. (in Arabic)

33. Wang Yali On the analysis of backhand attacking tactics of world famous male shake hands grip players, 10th Anniversary ITTF Sports Science Congress, University of Zagreb, Croatia, May, 2007.

34. X. W. Xiang Load Reach Of Multi-ball Means In Male Youth Footwork Training,

35. , http://www.globethesis.com, 2013. 
